\title{
General theorem of spatial coherence: application to three-dimensional imaging
}

\author{
Joseph Rosen and Amnon Yariv \\ California Institute of Technology, M.S. 128-95, Pasadena, California 91125
}

Received December 21, 1995; accepted May 16, 1996; revised manuscript received May 30, 1996

Three-dimensional imaging of incoherent light sources by the Michelson stellar interferometer is considered. When the interferometer's pinholes are arranged properly, its output result is equivalent to a two-dimensional Fourier hologram that stores information about the source object's three-dimensional intensity distribution. (C) 1996 Optical Society of America.

\section{INTRODUCTION}

The Michelson stellar interferometer ${ }^{1}$ (MSI) (also known as long-baseline interferometer) is used for measuring the shape of planar objects that emit quasi-monochromatic, spatially incoherent light. Its principle of operation is based on recording interference patterns in the far field obtained by a two-pinhole Young interference configuration. The fringes' visibility and phase versus the separation between the pinholes are equal to the complex degree of coherence in the plane of the pinholes. According to the Van Cittert-Zernike theorem, the complex degree of coherence is proportional to the Fourier transform of the source's (planar) intensity distribution. ${ }^{2}$ Thus measuring the complex degree of coherence versus pinhole separation and performing inverse Fourier transformation yield the image distribution of the source. The resolution of this imaging method is proportional to the maximum distance between the pinholes. Thus the resolution of an MSI with a maximum pinhole separation $D$ is approximately equal to the resolution of an ordinary imaging system with a full aperture of diameter $D$. This resolution property is the main advantage of the MSI as an imaging tool.

On the basis of the three-dimensional (3-D) paraxial generalization to the spatial coherence theorem that we derive here, we propose a method to measure the 3-D intensity distribution of source objects by the MSI, and we discuss the measurement limitations. We show that the MSI output result is equivalent to a two-dimensional (2-D) Fourier hologram that stores, in the form of spatial modulation, information about the source object's 3-D intensity distribution.

\section{THEORY}

A schematic diagram of the proposed MSI is shown in Fig. 1. A light source in the coordinate system $\left(x_{s}, y_{s}, z_{s}\right)$ illuminates from the far field two pinholes located in the coordinate system $(x, y, z)$. Without loss of generality, we assume that $z, z_{s}$ are on the same line. A volume el- ement source $d V_{m}$, with an amplitude $A_{m}(t)$, located a distance $R_{1}$ from one pinhole and $R_{2}$ from the other pinhole, illuminates them with spherical waves with a complex amplitude proportional to ${ }^{2} A_{m}\left(t-R_{i} / c\right) \exp [-j k(c t$ - $\left.\left.R_{i}\right)\right] / R_{i}$, where $i=1,2, c$ is the velocity of light, $k$ $=2 \pi / \lambda$, and $\lambda$ is the average wavelength. The complex degree of coherence, defined by the normalized time average of the product of the fields $E_{1}, E_{2}{ }^{*}$ at the pinholes' points, sampled at the same time, is

$$
\begin{aligned}
\mu\left(P_{1}, P_{2}\right) & =\frac{\left\langle E_{1}(t) E_{2}{ }^{*}(t)\right\rangle}{\left[\left\langle\left|E_{1}(t)\right|^{2}\right\rangle\left\langle\left|E_{2} *(t)\right|^{2}\right\rangle\right]^{1 / 2}} \\
& =\frac{\sum_{m} \sum_{n}\left\langle A_{m}(t) A_{n} *(t)\right\rangle \exp \left[j k\left(R_{1, m}-R_{2, n}\right)\right]}{\left[\sum_{m}\left\langle A_{m}(t) A_{m} *(t)\right\rangle \sum_{n}\left\langle A_{n}(t) A_{n} *(t)\right\rangle\right]^{1 / 2}} \\
& =\frac{\sum_{m}\left\langle A_{m}(t) A_{m} *(t)\right\rangle \exp \left[j k\left(R_{1, m}-R_{2, m}\right)\right]}{\sum_{m}\left\langle A_{m}(t) A_{m} *(t)\right\rangle} \\
& =I_{0}^{-1} \int I_{s}\left(\bar{r}_{s}\right) \exp \left[j k\left(R_{1}-R_{2}\right)\right] \mathrm{d}^{3} r_{s},
\end{aligned}
$$

where $I_{0}=\int I_{s}\left(\bar{r}_{s}\right) \mathrm{d}^{3} r_{s}$ and $I_{s}\left(\bar{r}_{s}\right)$ is the intensity per unit volume of the source; i.e., $I_{s}\left(\bar{r}_{m}\right) d V_{m}$ $=\left\langle A_{m}(t) A_{m} *(t)\right\rangle$. For each spherical wave we approximate the distance $R_{i}$ in the denominator by the average distance $R$. In this derivation we use the quasimonochromatic assumption ${ }^{2}$ [i.e., $\left\langle A_{m}\left(t-R_{1, m} / c\right) A_{m} *(t\right.$ - $\left.\left.\left.R_{2, m} / c\right)\right\rangle=\left|A_{m}\right|^{2}\right]$ and the complete incoherence of the source [i.e., $\left\langle A_{m}(t) A_{n} *(t)\right\rangle=\delta_{m n}\left|A_{m}\right|^{2}$ ]. In terms of the source's spectrum the quasi-monochromatic assumption is expressed by the condition $\Delta v \ll c R / \Delta r_{\max }^{2}$, where $\Delta v$ is the source's spectral bandwidth and $\Delta r_{\max }$ is the maximum separation between the pinholes.

Under the far-field assumption, that $R$ is much larger than the maximum size of the source, and the paraxial as- 
sumption, that $R$ is much larger than the maximum separation between the pinholes, $\left(R_{1}-R_{2}\right)$ is approximated as

$$
\begin{aligned}
R_{1}-R_{2}= & {\left[\left(R+z_{1}-z_{s}\right)^{2}+\left(x_{1}-x_{s}\right)^{2}\right.} \\
& \left.+\left(y_{1}-y_{s}\right)^{2}\right]^{1 / 2}-\left[\left(R+z_{2}-z_{s}\right)^{2}\right. \\
& \left.+\left(x_{2}-x_{s}\right)^{2}+\left(y_{2}-y_{s}\right)^{2}\right]^{1 / 2} \\
\cong & \Delta z+\frac{\hat{x} \Delta x+\hat{y} \Delta y}{R}-\frac{x_{s} \Delta x+y_{s} \Delta y}{R} \\
& +\frac{\Delta z\left(x_{s}{ }^{2}+y_{s}{ }^{2}\right)}{2 R^{2}}-\frac{z_{s}(\hat{x} \Delta x+\hat{y} \Delta y)}{R^{2}},
\end{aligned}
$$

where $\hat{a}=\left(a_{1}+a_{2}\right) / 2, \Delta a=a_{1}-a_{2}$, and $a$ stands for $x, y, z$. Substituting Eq. (2) into Eq. (1) yields

$$
\begin{aligned}
\mu\left(\bar{r}_{1}, \bar{r}_{2}\right)= & \frac{\exp (j k[\Delta z+(\hat{x} \Delta x+\hat{y} \Delta y) / R])}{I_{0}} \\
& \times \int I_{s}\left(\bar{r}_{s}\right) \exp \left\{\frac { - j 2 \pi } { \lambda } \left[\frac{x_{s} \Delta x+y_{s} \Delta y}{R}\right.\right. \\
& -\frac{\Delta z\left(x_{s}{ }^{2}+y_{s}{ }^{2}\right)}{2 R^{2}} \\
& \left.\left.+\frac{z_{s}(\hat{x} \Delta x+\hat{y} \Delta y)}{R^{2}}\right]\right\} \mathrm{d}^{3} r_{s} .
\end{aligned}
$$

Equation (3) is a generalization of the Van CittertZernike theorem to the case of correlation between two points at the paraxial zone that are illuminated by a 3-D source from the far field. We refer to it as the 3-D paraxial generalization to the spatial coherence theorem. Unlike a previous generalization, ${ }^{3}$ in which an observation from all 3-D directions is considered, ours is valid only for an observation from the paraxial regime. Also, we consider the two last phase terms in Eq. (3), which were apparently neglected in Ref. 3 .
The upper and lower pinholes, shown in Fig. 1, illuminate the lens with spherical waves emanating from points 1 and 2 , respectively, resulting in two plane waves at the back focal plane at angles $\varphi$ and $-\varphi$ to the lens's axis. When the contributions of all the quasi-monochromatic incoherent source points are summed, the intensity distribution in the back focal plane is

$$
\begin{aligned}
I\left(y_{0}\right)= & \int I_{s}\left(\bar{r}_{s}\right) \mid \frac{1}{R_{1}} \exp \left[j k\left(R_{1}+y_{0} \sin \varphi\right)\right] \\
& +\left.\frac{1}{R_{2}} \exp \left[j k\left(R_{2}-y_{0} \sin \varphi\right)\right]\right|^{2} \mathrm{~d}^{3} r_{s} \\
\cong & (1 / R)^{2} 2 I_{0}\left[1+\left|\mu\left(\bar{r}_{1}, \bar{r}_{2}\right)\right| \cos \left(2 k y_{0} \sin \varphi\right.\right. \\
& +\arg \{\mu\})],
\end{aligned}
$$

where $\mu\left(\bar{r}_{1}, \bar{r}_{2}\right)$ is the complex visibility function of the interference grating $I\left(y_{0}\right)$, and it is equal to the complex degree of coherence ${ }^{2}$ given in Eqs. (1) and (3). $\quad y_{0}$ is the transverse coordinate of the back focal plane, and we neglect the effect of the finite pinholes' apertures, assuming for simplicity that each of them is a mathematical point.

An MSI geometry that simplifies Eq. (3) is one where the pinholes' center of gravity is zero (i.e., $\hat{x}=\hat{y}=\hat{z}$ $=0$ ). In this case $\mu$ becomes a function of the coordinates $(\Delta x, \Delta y, \Delta z)$ :

$$
\begin{aligned}
\mu(\Delta x, \Delta y, \Delta z)= & \frac{\exp (j k \Delta z)}{I_{0}} \\
& \times \iint \hat{I}_{s}\left(x_{s}, y_{s}\right) \exp \left\{\frac { - j 2 \pi } { \lambda R } \left[\Delta x x_{s}\right.\right. \\
& \left.\left.+\Delta y y_{s}-\frac{\left(x_{s}{ }^{2}+y_{s}{ }^{2}\right) \Delta z}{2 R}\right]\right\} \mathrm{d} x_{s} \mathrm{~d} y_{s},
\end{aligned}
$$

where

$$
\hat{I}_{s}\left(x_{s}, y_{s}\right)=\int I_{s}\left(x_{s}, y_{s}, z_{s}\right) \mathrm{d} z_{s} .
$$

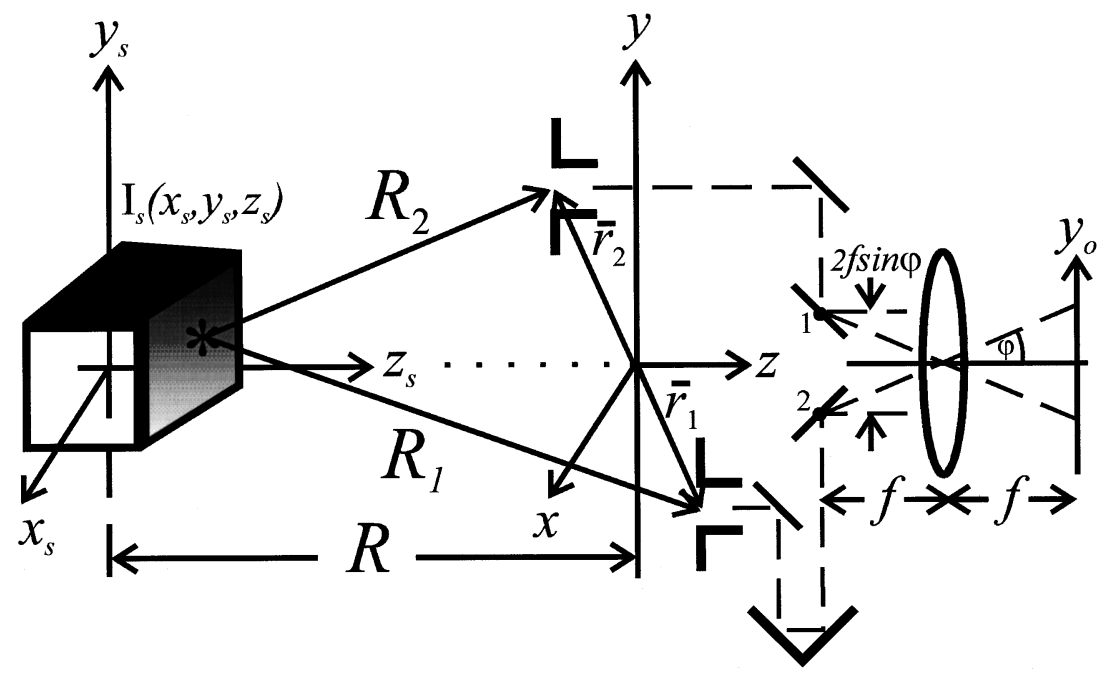

Fig. 1. Schematic system of the MSI for 3-D imaging. 
This expression for $\mu(\Delta x, \Delta y, \Delta z)$ is formally analogous to that describing coherent diffraction in a spherical lens system ${ }^{4}$ with focal length $R$ when an aperture with a field profile $\hat{I}_{s}\left(x_{s}, y_{s}\right)$ is displayed in the front focal plane. Note that the radial intensity distribution of the source can be reconstructed from measuring the coherence along the $z$ axis only. This is because $\mu(0,0, \Delta z)$ is a Fourier transform of $\hat{I}_{s}\left(\left|x_{s}{ }^{2}+y_{s}{ }^{2}\right|\right)$.

Another simplification results if the pinholes' axis is perpendicular to the $z$ axis $(\Delta z=0)$ : We fix one pinhole at the origin, and the pinholes are again illuminated by a 3 -D source. In this case $\mu$ becomes a 2-D function given by

$$
\begin{aligned}
\mu(x, y)= & C_{0} \int I_{s}\left(\bar{r}_{s}\right) \exp \left\{\frac { - j 2 \pi } { \lambda R } \left[x x_{s}+y y_{s}\right.\right. \\
& \left.\left.+\frac{\left(x^{2}+y^{2}\right) z_{s}}{2 R}\right]\right\} \mathrm{d}^{3} \bar{r}_{s},
\end{aligned}
$$

where $C_{0}=\exp \left[j k\left(x^{2}+y^{2}\right) / 2 R\right] / I_{0}$. This expression for $\mu(x, y)$ is also formally analogous to that describing a coherent diffraction in a spherical lens system. ${ }^{5}$ Suppose that a 3-D object with a profile equal to $I_{s}\left(\bar{r}_{s}\right)$ is located around the front focal plane $\left(z_{s}=0\right)$ of a lens with focal length $R$. When this object is illuminated by a plane wave propagating along $z$, the complex amplitude at the far (back) focal plane diffracted from this object has a distribution proportional to $\mu(x, y)$ given by Eq. (6). Note that the physics is different in this case, since this time both $I_{s}\left(\bar{r}_{s}\right)$ and $\mu(x, y)$ indicate coherent-field distributions. The complex field $\mu(x, y)$ in this case can be recorded in a 2-D hologram by interfering it with a second, coherent, reference, usually a plane wave at the recording plane. In our incoherent case the complex function $\mu(x, y)$ is recorded without any reference. The experimental recording of the complex function $\mu(x, y)$ can be accomplished as follows: The pinholes' plane of the MSI shown in Fig. 1 is arranged to be perpendicular to the $z$ axis. One pinhole is fixed at the origin, while the other is moved in a Cartesian grid. At each pinhole position $(x, y)$ a diffraction grating is generated in the MSI output. It should be noted that the location, spatial frequency, and orientation of these gratings depend on the geometry of the MSI and not on that of the source distribution. Therefore the only two parameters extracted from each grating (for each location of the moving pinhole) are its visibility and its phase. Completing the process along the full grid yields a sampled version of $\mu(x, y)$.

A reconstructed Fourier hologram field $\mu(x, y)$ of a coherent field $I_{s}\left(\bar{r}_{s}\right)$ can yield the (inverted) original field $I_{s}\left(\bar{r}_{s}\right)$ by propagating again through a lens to the vicinity of its back focal plane. On the basis of this analogy we achieve the reconstruction of the incoherent 3-D source by computing $u_{r}$ according to

$$
\begin{aligned}
u_{r}\left(x_{0}, y_{0}, z_{0}\right)= & \iint \mu(x, y) \exp \left\{\frac { j 2 \pi } { \lambda } \left[\frac{x x_{0}+y y_{0}}{L_{1}}\right.\right. \\
& \left.\left.+\frac{z_{0}}{2 L_{2}^{2}}\left(x^{2}+y^{2}\right)\right]\right\} \mathrm{d} x \mathrm{~d} y
\end{aligned}
$$

where $L_{1}, L_{2}$ are reconstruction constants. In the case of an optical reconstruction, $\mu(x, y)$ is placed in the front focal plane of a lens with focal length $f$. It is straightforward to show that Eq. (7) describes the complex-field distribution around the back focal plane $\left(x_{0}, y_{0}, z_{0}=0\right)$ if $L_{1}=L_{2}=-f$, and $u_{r}$ is multiplied by a linear phase factor of $z_{0}$, which is eliminated by any intensity detector.

In order to see that the original object is indeed reconstructed by the process described by Eq. (7), let us describe the 3-D source as a discrete collection of slices each of which has a thickness of a resolution element $\Delta z_{s, \min }$. Equation (6) is rewritten for this case as follows:

$$
\begin{aligned}
\mu(x, y)= & C_{0} \sum_{n} \Delta z_{s, \min } \iint I_{s}\left(x_{s}, y_{s} ; z_{s, n}\right) \\
& \times \exp \left\{\frac { - j 2 \pi } { \lambda R } \left[x x_{s}+y y_{s}\right.\right. \\
& \left.\left.+\frac{\left(x^{2}+y^{2}\right) z_{s, m}}{2 R}\right]\right\} \mathrm{d} x_{s} \mathrm{~d} y_{s} .
\end{aligned}
$$

Substitute $\mu(x, y)$, given by Eq. (8), into Eq. (7) and solve for the field $u_{r}$ at the plane $\bar{z}_{0}=\bar{z}_{s} L_{2}{ }^{2} / R^{2}$, where $\bar{z}_{s}$ indicates some arbitrary transverse plane of the original 3-D object space. The reconstructed pattern at this plane is

$$
\begin{aligned}
u_{r}\left(x_{0}, y_{0} ; \bar{z}_{0}\right) \\
=\Delta z_{s, \min } I_{s}\left(\frac{-x_{0} R}{L_{1}}, \frac{-y_{0} R}{L_{1}} ; \frac{-\bar{z}_{0} R^{2}}{L_{2}{ }^{2}}\right) \\
\quad+\sum_{z_{s, n} \neq R^{2} \bar{z}_{0} / L_{2}{ }^{2}} \Delta z_{s, \min } \iint I_{s}\left(x_{s}, y_{s} ; z_{s, n}\right) \\
\quad \times \frac{\lambda R^{2} L_{2}^{2}}{z_{s, n} L_{2}^{2}-R^{2} \bar{z}_{0}} \\
\quad \times \exp \left\{\frac { - j \pi R ^ { 2 } L _ { 2 } ^ { 2 } } { \lambda ( z _ { s , n } L _ { 2 } ^ { 2 } - R ^ { 2 } \overline { z } _ { 0 } ) } \left[\left(\frac{x_{0}}{L_{1}}+\frac{x_{s}}{R}\right)^{2}\right.\right. \\
\left.\left.\quad+\left(\frac{y_{0}}{L_{1}}+\frac{y_{s}}{R}\right)^{2}\right]\right\} \mathrm{d} x_{s} \mathrm{~d} y_{s} .
\end{aligned}
$$

The first term in Eq. (9) is the scaled reconstruction of the original object at the plane $z_{s}=\bar{z}_{s}=R^{2} \bar{z}_{0} / L_{2}{ }^{2}$. The second term is the unfocused distribution obtained from all the object points that are not on the plane $z_{s}=\bar{z}_{s}$. Repeating the procedure of Eq. (9) for each transverse plane $z_{0}$, the original pattern of the corresponding plane, $z_{s}=R^{2} z_{0} / L_{2}{ }^{2}$, is focused. The unfocused term in Eq. (9), which involves the summation, prevents us from obtaining a perfect reconstruction of the original object $I_{s}\left(\bar{r}_{s}\right)$. We should note, however, from the rigorous holographic analogy of Eq. (6), that the field $u_{r}\left(x_{0}, y_{0}, z_{0}\right)$ is that which would be seen by an observer when the 3-D object was illuminated by a coherent field. This is the same field that results from a reconstruction of a hologram of $I_{s}\left(\bar{r}_{s}\right)$, which is the basis of holographic 3-D imaging. In this sense we can claim to image the 3-D object $I_{s}\left(\bar{r}_{s}\right)$.

The magnification of this imaging process is $L_{1} / R$ in the transverse dimension and $\left(L_{2} / R\right)^{2}$ along the $z$ axis. The minimum depth distance that can be resolved in this 
(a)

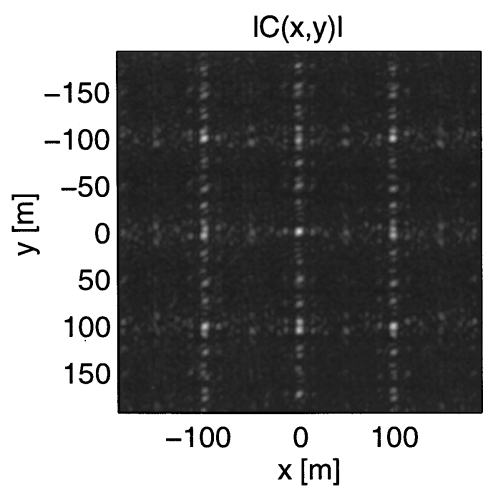

(c)

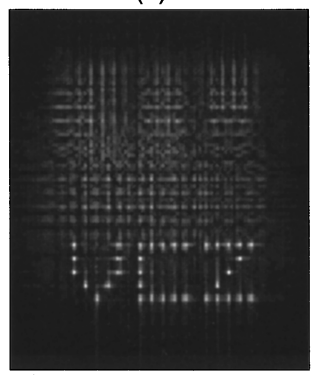

$z=-50$ (b)

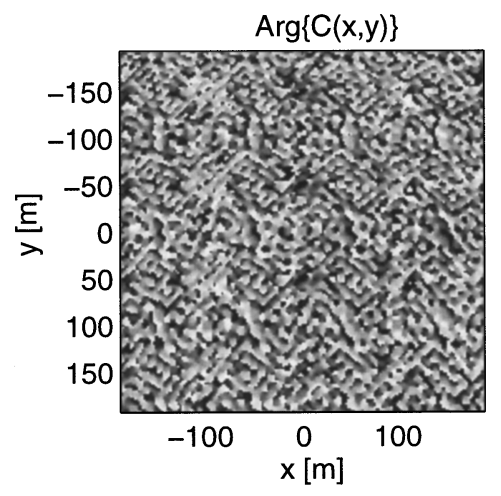

(e)

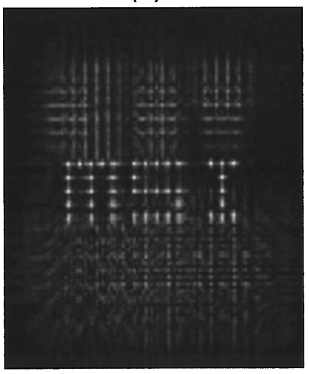

$z=50$

Fig. 2. Computer simulation of the MSI. The magnitude (a) and the phase (b) of the 2-D complex visibility function were calculated from the interference MSI's gratings. (c)-(e) The reconstruction of the hologram shown in (a) and (b) at three different planes along the $z$ axis. In each plane only one abbreviation is in focus, indicating its original location in the object space.

case is $\Delta z_{s, \text { min }} \approx 2 \lambda R^{2} / r_{\text {max }}^{2}$, where $r_{\text {max }}=\left(x_{\text {max }}^{2}+y_{\text {max }}^{2}\right)^{1 / 2}$ and $\left(x_{\max }, y_{\max }\right)$ are the maximum distances reached by the moving pinhole. On the other hand, the minimum planar distance that can be resolved in the target is $\left(\Delta x_{s, \min }, \Delta y_{s, \min }\right) \approx\left(\lambda R / x_{\max }, \lambda R / y_{\max }\right)$. Assuming that the observed object is limited by a box with sides of length $\left(X_{S}, Y_{S}, Z_{S}\right)$, the step size of the pinhole $\left(\delta x_{\max }, \delta y_{\max }\right)$ should be no larger than $\lambda R^{2}\left[\left(x_{\max } Z_{s}\right.\right.$ $\left.+R X_{s}\right)^{-1}$, $\left(y_{\max } Z_{s}+R Y_{s}\right)^{-1}$ ], in order for the whole object to enter into the MSI's field of view.

\section{SIMULATION RESULTS}

As an example we take three abbreviations as our quasimonochromatic incoherent homogeneous light source. Each of the three abbreviations is positioned at a different location along the $z_{s}$ axis, and is composed from an incoherent collection of point sources of spherical waves. Our simulation is based on the first two lines of Eq. (4) for calculating the degree of coherence and on Eq. (7), for calculating the reconstructed pattern. Without any further approximation, the following results are also used as a verification of Eq. (6).

We simulated the MSI operation with the parameters $R=10^{5} \mathrm{~m}, \quad \lambda=10 \mu \mathrm{m}, \quad\left(x_{\max }, y_{\max }\right)=(192,192) \mathrm{m}$, $\left(X_{S}, Y_{S}, Z_{S}\right)=(0.2,0.2,22) \mathrm{m}, \quad\left(\delta x_{\max }, \delta y_{\max }\right)=$ $(2,2) \mathrm{m}$. The results are shown in Fig. 2. In our simulated MSI we assume that the gratings are all one dimensional, with the same frequency and orientation. The only important information contained in this collection of gratings is their visibility and phase. These two parameters are measured in each grating, and the resulting 2-D visibility and phase distribution are shown in Figs. 2(a) and $2(\mathrm{~b})$, respectively. Each value $(i, j)$ in this matrix indicates the visibility, Fig. 2(a), and phase, Fig. 2(b), of the grating obtained when the moving pinhole is in the $(i, j)$ location. Finally, the reconstruction results in three planes along the $z_{0}$ axis are shown in Figs. 2(c)2(e). These results are obtained by calculating Eq. (7) for three different values of $z_{0}$. At each plane a different abbreviation is focused; thus reconstruction of the object in the $3-\mathrm{D}$ space is possible.

\section{CONCLUSION}

We have proposed and demonstrated a new method of synthetic-aperture incoherent holography. With this method we are able to measure the complex visibility function containing 3-D information about the incoherent source. This 3-D distribution can be reconstructed in the same sense as a coherently recorded hologram. The system has a synthetic-aperture width equal to double the maximum separation between the pinholes, whereas the actual aperture consists only of the apertures of the two pinholes. This property gives to the proposed method an advantage over other incoherently recorded holograms. ${ }^{6}$

\section{ACKNOWLEDGMENTS}

This research was supported by the U.S. Army Research Office and the Advanced Research Projects Agency. 


\section{REFERENCES}

1. W. J. Tango and R. Q. Twiss, "Michelson stellar interferometry," in Progress in Optics, E. Wolf, ed. (Elsevier, New York, 1980), Vol. 17, pp. 239-277.

2. M. Born and E. Wolf, Principles of Optics, 4th ed. (Pergamon, Oxford, 1970), Chap. 10, p. 491; J. W. Goodman, Statistical Optics, 1st ed. (Wiley, New York, 1985), Chap. 5, p. 157.

3. W. H. Carter and E. Wolf, "Correlation theory of wavefieldsgenerated by fluctuating, three-dimensional, primary, scalar sources," Opt. Acta 28, 227-244 (1981).

4. C. W. McCutchen, "Generalized source and the Van Cittert-Zernike theorem: a study of the spatial coherence required for interferometry," J. Opt. Soc. Am. 56, 727-733 (1966); J. Rosen and A. Yariv, "Longitudinal partial coherence of optical radiation," Opt. Commun. 117, 8-12 (1995).

5. A. Yariv, Optical Electronics, 4th ed. (Saunders, Philadelphia, Ca., 1991), App. E, p. 705.

6. A. W. Lohmann, "Wavefront reconstruction for incoherent objects," J. Opt. Soc. Am. 55, 1555-1556 (1965); G. Sirat and D. Psaltis, "Conoscopic holography," Opt. Lett. 10, 4-6 (1985). 